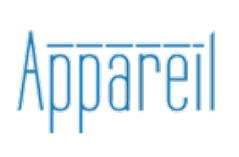

Appareil

23 | 2021

Poïétique du jeu vidéo

\title{
The Stanley Parable : le joueur contre le narrateur
}

\section{Douglas Hoare}

\section{(2) OpenEdition}

\section{Journals}

\section{Édition électronique}

URL : http://journals.openedition.org/appareil/3954

DOI : 10.4000/appareil.3954

ISSN : 2101-0714

Éditeur

MSH Paris Nord

\section{Référence électronique}

Douglas Hoare, «The Stanley Parable : le joueur contre le narrateur », Appareil [En ligne], 23 | 2021, mis en ligne le 31 mars 2021, consulté le 02 avril 2021. URL : http://journals.openedition.org/appareil/3954 ; DOI : https://doi.org/10.4000/appareil.3954

Ce document a été généré automatiquement le 2 avril 2021.

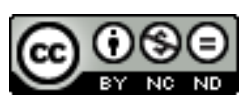

Appareil est mis à disposition selon les termes de la Licence Creative Commons Attribution - Pas d'Utilisation Commerciale - Pas de Modification 4.0 International. 


\title{
The Stanley Parable : le joueur contre le narrateur
}

\author{
Douglas Hoare
}

1 The Stanley Parable, développé par le studio Galactic Cafe et paru en 2013, fait intervenir au cœur de son mécanisme de jeu une figure littéraire par excellence : le narrateur. La chose n'est pas courante et mérite d'être soulignée. Bien des jeux vidéo accordent une place non négligeable à la narration et situent le déroulement de la partie dans le cadre d'un récit ${ }^{1}$, mais bien peu mettent en scène un narrateur au cours du jeu, pour la bonne et simple raison que la suite programmée des évènements, telle qu'elle est planifiée dans le scénario et réalisée dans la partie, se suffit à elle-même, n'a besoin d'être portée par aucune voix. Le cours de l'histoire se manifeste de façon implicite dans l'agencement des situations auxquelles le joueur est confronté.

2 Par ailleurs, la narration vidéoludique tente de faire passer, durant le cours de la partie, le fil d'un scénario pré-écrit pour une suite de décisions appartenant au joueur, pour compenser son inscription au sein d'une trame qu'il ne maîtrise pas. Dans ce que nous appellerons à notre tour un jeu narratif (pour reprendre l'expression de MarieLaure Ryan et Anne-Laure Rebreyend ${ }^{2}$ ), l'interactivité propre au jeu vidéo a une portée sur le récit. Dans de tels jeux, il ne s'agit pas simplement de perdre ou gagner; le dispositif contient la promesse que l'action du joueur est déterminante pour la suite de l'histoire. Elle est au moins présentée comme telle. C'est à travers les situations de jeu, et donc les exploits du joueur, que le récit peut avoir lieu.

Cela laisse donc, en dehors des étapes inévitables du récit, une certaine liberté au sujet humain de l'expérience : celle de mener son avatar comme bon lui semble durant les séquences de jeu et donc de concourir à la fois à la mise en scène et à la narration de son épopée. En incarnant le protagoniste, en l'animant, il a le sentiment de produire son propre récit et en devient de fait le point de focalisation. Il éprouve les événements de première main, sans la voix d'un autre, sans médiation autre que celle du programme. L'interactivité lui laisse une certaine latitude qui varie selon les jeux vidéo. Le lecteur parait, en comparaison de ce type de joueur, être une figure plus passive et surtout moins physiquement engagée dans la production d'une histoire. Du reste, les 
héros en littérature sont plus dociles que n'importe quel avatar: "Imaginez Shakespeare devoir écrire Hamlet sans contrôler tous les personnages ${ }^{3} ! »$.

4 Si un jeu vidéo intégrait à son dispositif un narrateur omniscient, dictant les moindres faits et gestes de l'avatar, prétendant connaître ses émotions et anticiper ses réactions, il en résulterait pour le joueur un désagréable sentiment d'étouffement : il serait ainsi projeté dans la position de simple exécutant. Il deviendrait alors aussi peu libre qu'un personnage de roman ou de théâtre, contraint à tout instant de suivre une conduite décidée par un autre. C'est ce que fait The Stanley Parable. En revanche, le jeu ajoute une porte de sortie : si la contrainte de la narration est personnifiée, c'est pour que l'on puisse lui désobéir. Lorsque Stanley, avatar du joueur, arrive devant deux portes, la voix lui intime de prendre celle de gauche. Libre à lui de prendre celle de droite. Une lutte s'instaure alors entre le joueur et le narrateur pour le contrôle de l'histoire.

Cette lutte n'est-elle pas à l'image de l'ambivalence entre poïèse et esthèse ${ }^{4}$ qui structure toute expérience vidéoludique? N'est-elle pas l'expression imagée de la lutte intérieure que connaît tout joueur? À travers elle, The Stanley Parable semble tracer les limites du contrôle que ce dernier peut exercer sur la narration et fait de ce contrôle l'enjeu même de la partie. C'est un jeu avec la narration que l'on nous propose. La parabole évoquée dans le titre est celle de la condition du joueur et de sa liberté réelle ou fantasmée. Son déploiement permet de jauger la mesure dans laquelle le joueur est libre de se faire narrateur.

\section{L'histoire de Stanley}

6 D'emblée, l'écran d'accueil nous avertit de la mise en abyme. Il se répète à l'infini dans un ordinateur de bureau se détachant sur un mur gris. Puis, l'écran devient noir. C'est une voix d'homme que l'on entend: "C'est l'histoire d'un homme nommé Stanley "». Nous ne jouons pas, nous écoutons. Des scènes défilent alors, au rythme du texte qui nous est lu. Le narrateur nous raconte l'histoire d'un employé, l'employé 427 d'une entreprise anonyme qui, assis au bureau 427 , se contente pour tout travail de presser les touches de son clavier quand son écran le lui ordonne. Des signaux lumineux lui indiquent les boutons à enclencher, le temps qu'il faut les presser, etc. Cet effort robotique ne décourage pas notre employé fantôme ${ }^{6}$. Le narrateur nous confie même qu'il est heureux ; jusqu'au jour où, sans raison apparente, les ordres ne viennent plus. Plongé dans le plus grand désarroi, nous dit encore la voix alors même que le jeu commence et que nous pouvons prendre contrôle de notre avatar, Stanley décide de sortir de son bureau à la recherche de ses collègues, qui ne lui ont donné aucun signe de vie depuis plus d'une heure.

7 Tout au long de cette séquence empruntant son ton au théâtre de l'absurde, c'est la voix du narrateur qui domine, nous guide et assure la transition entre l'introduction du jeu et le début de la partie. Nous constatons avec étonnement que cette voix, loin de se taire pour laisser place à l'action, continue son invraisemblable monologue, comme si nous étions encore au stade du récit, comme s'il fallait encore planter le décor. Mieux, elle semble anticiper nos agissements, et par là, les diriger. C'est encore elle qui exprime les pensées de notre pantin resté muet qui est, à ce moment, un parfait étranger. Stanley, fort étonné (si l'on en croit le narrateur) de trouver les bureaux voisins déserts, se dirige ensuite vers la salle de réunion. 
8 Le joueur en charge de Stanley n'a d'autre choix que de s'exécuter. L'étendue des talents de notre avatar ne permet rien d'autre. Conformément à ce qui semble être sa spécialité, Stanley peut marcher et porter son regard dans toutes les directions, ouvrir des portes ou appuyer sur des boutons, ce qui fait dire à Tristan Bera que The Stanley Parable est « une sorte de simulation de marche ${ }^{7}$ ». Cette absence apparente de gameplay a quelque chose de frappant. D'habitude, les jeux vidéo donnent à nos mouvements infimes une portée supérieure; mais ici, lorsque l'on presse la touche servant à actionner un élément du décor, le son d'une touche de clavier enfoncée retentit de l'autre côté de l'écran. Cet écho nous nargue, redouble stupidement notre geste au lieu de lui conférer une dimension spectaculaire. Ce geste identique établit instantanément le lien entre les identités du joueur et de Stanley. Les deux font la même chose, ont pour ainsi dire - le même travail, à tel point qu'il paraît difficile de considérer au départ The Stanley Parable comme un jeu.

9 La chose serait assurément plus supportable si l'on nous laissait déambuler où bon nous semble, ou si l'on nous donnait quelque chose à voir. Seulement, dans les premiers instants, il n'y a qu'un chemin tout tracé dans le décor gris et si peu dépaysant d'une entreprise du secteur tertiaire, avec sa moquette, ses imprimantes, ses dossiers, ses tasses de café, ses ordinateurs et ses plantes vertes. Sans compter, nous l'avons dit, que le narrateur persiste. En devenant Stanley, en animant notre avatar, nous nous sommes mis à sa merci. Il dicte l'histoire que nous sommes censés prendre en main, prédit nos moindres faits et gestes, décrit nos états d'âme.

Cette omniscience manifeste est habituelle dans une nouvelle ou un roman, mais est extrêmement déconcertante dans un jeu vidéo. La vision "par derrière ", propre au récit classique d'après Tzvetan Todorov, fait son irruption dans l'univers vidéoludique. Dans The Stanley Parable également, "le narrateur en sait davantage que son personnage. Il ne se soucie pas de nous expliquer comment il a acquis cette connaissance : il voit à travers les murs de la maison aussi bien qu'à travers le crâne de son héros ${ }^{8}$.» Ce type de narration entre en contradiction directe avec l'interactivité du support, puisqu'elle ne laisse pas le joueur libre du rapport qu'il établit avec lui.

11 Le joueur contrôle Stanley, certes, mais Stanley est lui-même contrôlé par le narrateur. Stanley possède à la fois l'inconsistance d'un avatar de jeu vidéo - nous ne savons rien de lui, il n'est que ce qu'il fait - et l'autonomie d'un personnage de roman - il semble posséder un rôle précis dans une histoire écrite par un autre, auquel on ne peut se soustraire. Il n'est plus le jouet du joueur, mais de l'intrigue. Non seulement Stanley est un avatar impuissant, mais nous ne décidons pas de ce qu'il fait. Pourtant, nous le dirigeons encore.

12 Nous retrouvons ici, dans le domaine du jeu et non du travail, tous les traits de l'aliénation au sens de Marx: "l'idée d'aliénation implique une pensée du possible puisque c'est seulement en référence à l'idée d'un possible non réalisé que l'activité ou la situation peut être dite "aliénée" ; et c'est cet avortement d'une possibilité de départ au sein d'une réalité qui pourtant la porte que seul le concept d'aliénation permet d'évoquer9." Dans ces premiers moments, en annulant aussi bien les vertus dépaysantes du support que la mise en scène de toute-puissance qu'il convoque habituellement et jusqu'à la possibilité de jouer, The Stanley Parable semble renoncer dans un acte de parfait sabotage à tous les possibles du jeu vidéo.

13 Notre désir de jouer est donc parfaitement contrecarré, jusqu'au moment où, guidé par la voix, nous arrivons au dilemme fondateur de l'expérience. Une pièce et deux portes 
s'offrent à nous. Le narrateur dirige Stanley vers celle de gauche, tandis que le jeu laisse la possibilité d'emprunter celle de droite. Ce qui était indissociable dans les premiers instants, à savoir l'agencement programmé des espaces - autrement dit les possibilités concrètes d'exploration - et la voix du narrateur, se trouve ici sciemment dissocié. C'est un retournement subtil, dont le joueur ne peut saisir immédiatement la mesure. Il s'y joue cependant un renversement complet de la partie.

Le narrateur, qui jusqu'alors n'était que la personnification du cours obligé des choses, de cette entité souterraine qui est dans le jeu vidéo l'agent de la fatalité extérieure, à savoir le programme, se trouve par là potentiellement réduit au rang de simple personnage. Cette fatalité si pesante peut être enfin contredite. Tout dépend alors du choix du joueur. Il est comme l'âne de Buridan, tiraillé non pas entre la faim et la soif, mais entre une histoire cohérente et le plaisir de jouer. Alors que, dans la plupart des jeux vidéo, le joueur obtient la première par le second, The Stanley Parable dissocie la synthèse opérée habituellement, place les deux termes du problème aussi loin l'un de l'autre que possible, sous la forme de ces deux portes.

Si le joueur choisit la porte de gauche, tout continue comme avant. Le narrateur dicte son histoire, que Stanley exécute avec la plus grande docilité. Toutefois, si ce dernier emprunte la porte de droite, le narrateur perd de fait son omniscience. Il se passe quelque chose que la voix n'avait pas prévu. Elle ne parvient plus à pénétrer comme au départ la conscience de son protagoniste. Pour reprendre les termes de Todorov, d'une vision "par derrière ", nous passons à une vision «avec " - où le narrateur n'en sait pas plus que les personnages -, voire à une vision «du dehors ${ }^{10} »-$ où le narrateur en sait moins que les personnages.

Par un jeu de vases communicants, la perte de contrôle du narrateur entraîne un regain de contrôle pour le joueur. Ce regain permettrait enfin au joueur de diriger son personnage, de le faire agir comme bon lui semble dans les limites techniques induites par le programme. Le sujet humain pourrait de nouveau concourir à la narration et à la mise en scène de l'expérience, esquisser chez l'avatar un caractère qui dépendrait du sien, ajouter une touche de personnalité au cours des évènements. Il quitterait la passivité d'un spectateur agissant, parfaitement conditionné, et s'affirmerait ainsi pleinement en tant que joueur. Prendre la porte de droite est donc un acte de guerre narratif, qui ouvre une lutte entre le joueur et le narrateur pour le contrôle de Stanley et de son histoire.

\section{La parabole}

Cette liberté nouvellement offerte est des plus séduisantes. Elle tient simplement à ce refus augural, à cette désobéissance, triviale en apparence. La liberté du joueur s'affirme comme une liberté négative ${ }^{11}$ au sens d'Isaiah Berlin. Celle-ci consiste à se défaire ou à refuser toute immixtion extérieure relativement à ses choix - c'est une aire de libération de l'intervention d'autrui. Cette conception de la liberté s'inscrit dans la lignée des penseurs libéraux anglo-saxons et c'est cette conception que The Stanley Parable met en jeu - au sens propre comme au figuré.

Tout nous porte vers ce refus, vers la conquête de cette liberté négative, en particulier l'habitude du joueur aguerri de pouvoir en disposer dès le départ. Il va de soi que le jeu vidéo est une expérience programmée et qu'il s'agit là probablement davantage d'un sentiment de liberté que d'une liberté réelle. Le joueur de jeu vidéo exerce son libre- 
arbitre au sein d'une expérience parfaitement conditionnée ; mais lorsque The Stanley Parable rend explicite ce conditionnement par la figure du narrateur, interdisant jusqu'au dilemme des portes tout exercice de ce libre-arbitre, le dispositif incite le joueur à s'emparer de cette liberté dès qu'il en a l'occasion. Cependant, si malgré ses attraits, Stanley délaisse la porte de droite, porte de la liberté ou de la rébellion, le narrateur et le jeu lui réservent un dénouement parfaitement heureux.

Si l'ordre est respecté et que Stanley, c'est-à-dire le joueur, se soumet à la volonté du narrateur, à quoi assistons-nous? Ne trouvant personne dans l'entreprise, Stanley se dirige vers le bureau de son patron pour constater qu'il est, lui aussi, absent. La voix lui donne alors tout haut le code secret d'une porte dérobée, non sans insister en bon narrateur omniscient sur le fait que le pauvre Stanley ne peut qu'ignorer ce secret. Par le plus grand des hasards, nous dit-on, le joueur saisit les bons chiffres et la porte s'ouvre. Elle mène à un centre de contrôle des esprits - a mind control facility. C'est d'abord un panoptique, une salle couverte d'écrans où l'on surveille chacun des employés. Chemin faisant, la voix nous apprend la révolte intérieure de Stanley, sa colère face à l'idée que sa vie pourrait être sous le contrôle d'un autre. Nous voilà ensuite dans une salle de contrôle, où les émotions et le comportement de chaque employé peuvent être sollicités par la pression d'un bouton. Résolument révolté d'après le narrateur, Stanley est décidé à détruire cette machine infernale. Il se fraie un chemin jusqu'à l'alimentation centrale de l'appareil. Deux boutons s'offrent à lui, portant les indications d'usage : ON et OFF. La voix nous révèle que Stanley estime qu'il est de son devoir de mettre fin à tout cela. Toujours docile, obéissant à l'ordre final du narrateur, le joueur coupe le courant en pressant la touche adéquate. Tout devient noir. Stanley, nous dit-on, a vaincu la machine. "Il se délivra du contrôle d'autrui ${ }^{12}$. ». Débarrassé de ce contrôle qui l'enchaînait depuis si longtemps, Stanley retrouve le chemin de la liberté. Une porte gigantesque s'ouvre sur un paysage bucolique. Un sentier entre des arbres l'attend, figurant le seul coin de nature du jeu rencontré jusqu'alors. "C'était exactement la façon, à cet instant précis, dont les choses devaient se passer. Et Stanley était heureux ${ }^{13} . »$.

20 Impossible de ne pas sentir l'inquiétante ironie de cette fin surprenante. Le dispositif vidéoludique est aligné sur celui d'une nouvelle - au sens littéraire, techniquement assistée, requérant du joueur une participation minimale. La contradiction des moyens et des fins est tendue à l'extrême : c'est en se soumettant absolument au narrateur, à cette voix qui lui dicte à tout moment sa conduite, que Stanley se libère. C'est en ne prenant aucune part active à l'histoire que celle-ci se déroule sans accroc. C'est en subissant un contrôle absolu que le joueur délivre son personnage. C'est en se soumettant en toute occasion au fantôme divin du narrateur qu'on lui présente l'image - ou devrait-on dire un cliché des plus éculés - de la liberté. En outre, que penser de ces derniers mots, qui sont aussi ceux de l'introduction et dont la répétition semble annuler tout le chemin parcouru jusque-là ?

$21 \quad$ Et si Stanley n'avait jamais quitté son bureau ? Et s'il était toujours au travail ? Et s'il avait suivi les consignes lumineuses de son écran, lui indiquant quelles touches presser et dans quel ordre? Comment ne pas voir que si Stanley quitte son poste et se détourne de l'écran, le joueur reste derrière le sien pour s'adonner à une activité qui, abstraction faite de toute dimension ludique, se rapporte exactement à celle de son antihéros? C'est bien la condition de joueur de jeu vidéo qui est l'objet de la parabole de Stanley. 
Avec son ton sarcastique absolument moderne, The Stanley Parable s'attaque aux codes et aux canons de l'industrie et va jusqu'à les déconstruire pour prendre parti contre l'expérience qu'ils proposent. Cette fin nous révèle pourquoi il a fallu faire intervenir dans l'espace du jeu vidéo la figure littéraire du narrateur et pourquoi les autres jeux se gardent bien d'agir de la sorte. Sous l'effet de la figuration, la contrainte impersonnelle du programme devient l'autorité de ce personnage. Cette personnification d'un processus est là pour permettre la prise de conscience d'un mécanisme habituellement refoulé, rejeté d'autant plus facilement dans les tréfonds de la conscience qu'il se confond absolument avec le monde du jeu.

Dans la plupart des jeux, le programme est un dieu souterrain - il ordonne les situations et les événements. Dans The Stanley Parable, c'est le rôle du narrateur, c'est le rôle de la voix. Cette dissociation, alliée à l'absence totale de gameplay, déconstruit l'expérience du jeu vidéo. Cette scission donne à voir son fonctionnement essentiel : des signaux lumineux d'un côté de l'écran, des opérations techniques de l'autre. S'ajoute à cela la trame des événements présentée dans le cas que nous avons examiné.

Selon le Littré, une parabole est une «allégorie qui renferme quelque vérité importante. La parabole a deux parties, le corps et l'âme; le corps est le récit de l'histoire qu'on a imaginée ; et l'âme, le sens moral ou mystique, caché sous les paroles ou récit ${ }^{14}$. » L'âme de la parabole donnant son titre au jeu, la liberté, pourrait s'énoncer en ces termes:

Plus le joueur obéit aux stimulations du programme, plus il se conforme sans retard aux ordres de la machine, plus il contraint ses gestes à suivre la cadence imposée et plus son personnage triomphe. Le héros qui le représente accumule les victoires et multiplie les exploits. Autrement dit, plus l'avatar est libre, plus le joueur est esclave. En jouant à un jeu vidéo, on échange une soumission réelle contre une liberté simulée ${ }^{15}$.

Pour que l'avatar conquière son bonheur, le joueur doit renoncer à toute liberté, devenir l'exécutant d'une histoire à laquelle il ne fait que se soumettre.

\section{La porte de droite}

Pour tenter d'échapper à ce troc, le joueur peut emprunter la porte de droite. Il se préserve alors de l'influence du narrateur et sa conduite se teinte d'un semblant de libre-arbitre. Par ce geste simple, le sujet humain tente de reprendre en main son histoire. La première désobéissance est acceptée avec agacement par le narrateur. Ce n'est encore qu'un détour. Néanmoins, elle en appelle d'autres. Au fur et à mesure, la voix se fait plus fébrile. Toute affirmation de Stanley, qui est en fait une affirmation du joueur, est une négation de son pouvoir. La liberté négative, manifestée par le refus, tente de se muer en liberté positive ${ }^{16}:$ la question n'est plus simplement de se soustraire, de ne plus être entravé dans ses choix, mais de devenir son propre maître. Il s'agit d'écrire son histoire. Ce faisant, les possibles du jeu s'ouvrent. C'est à ce moment que la partie commence.

The Stanley Parable change alors de statut. Ce n'est plus une simple narration interactive, c'est désormais un jeu vidéo, même si Stanley, notre avatar, est toujours aussi infirme. Nous nous heurtons, il est vrai, de mille façons au même mur, à l'impossibilité de jouer de façon attendue - dans le cas où le jeu mobiliserait notre adresse ou notre réflexion, nous donnerait des ennemis à vaincre ou des énigmes à 
résoudre. Cependant, les différents embranchements narratifs sont autant de lieux et de fins à découvrir, de même que le monologue du narrateur prend différents accents, mène à différentes questions, révèle, de façon kaléidoscopique dans la juxtaposition apparente de trames non résolues, la véritable forme du jeu.

La narration est au cœur de la lutte de pouvoir qui structure la partie. C'est autour de sa maîtrise que se constitue la dialectique du maitre et de l'esclave ${ }^{17}$ entre le narrateur et Stanley. L'action du joueur devient une critique en actes de l'harmonie préétablie du récit. Le gameplay intégrant cette critique à son dispositif, le narrateur est réduit à l'impuissance par le programme, qui permet au joueur de suivre ou non les ordres de la voix. Par conséquent, la cohérence du récit vole en éclats. Fins abruptes, éternels recommencements, intermèdes musicaux, téléportation de Stanley dans un décor de Minecraft ${ }^{18}$ ou de Portal ${ }^{19}$, sans parler des textures qui se désagrègent ou des salles encore en construction, voilà ce que le joueur s'acharne à découvrir en cherchant à épuiser le jeu. Dans sa volonté d'intégrer le négatif, The Stanley Parable va jusqu'à simuler le bug.

Chacun de nos coups d'aile libre entraîne dans son sillage son lot d'imprévus ou de catastrophes. La promesse de l'interactivité est ici maintenue, mais négativement. L'interactivité nous promet d'exercer une influence sur le cours des choses, simule la portée de nos gestes. Quand le dispositif le permet, le joueur peut donc préférer un geste beau ou un geste efficace, exécuter le geste qu'il aurait envie de voir ou le geste requis par le programme. C'est un conflit interne qui tient au désir prédominant chez lui : celui de la performance ou celui de la portée esthétique de ses actes. Parfois, selon le jeu, l'efficacité et la cohérence esthétique sont liées. Dans un jeu d'aventure par exemple, notre action s'intègre au récit, affirme la cohérence du monde et de l'expérience qui, en retour, nous incite à agir derechef. Ici, c'est au contraire la destruction de l'univers du jeu et la révélation de sa fragilité qui est, en somme, notre récompense. L'action de Stanley n'apporte que le chaos - qu'il soit scénaristique ou visuel; ou, plus précisément, le simulacre d'un chaos, l'apparence d'une destruction cathartique.

The Stanley Parable permet au joueur de retrouver à l'écran le plaisir enfantin du saccage, d'une façon parfaitement circonscrite par le code informatique. Le jeu donne libre cours à un certain sadisme. La fragmentation du récit et du décor marque le passage du joueur, sont la preuve de sa puissance, exercée d'abord au grand dam du narrateur - mais toujours avec la bénédiction du programme.

On pourrait considérer que le joueur est placé dans la situation du briseur de jeu décrite par Johan Huizinga :

[Le] briseur de jeu est tout autre que le faux joueur. Ce dernier feint de jouer le jeu.

[...] La communauté lui pardonne plus volontiers qu'au précédent, car celui-ci détruit leur univers. En se dérobant, il découvre la valeur relative et la fragilité de cet univers. Il enlève au jeu l'illusion, inlusio, littéralement "entrée dans le jeu ", mot chargé de signification. Aussi doit-il être éliminé, car il menace l'existence de la communauté joueuse ${ }^{20}$.

Le narrateur tient au départ le rôle de la communauté - c'est lui qui nous reproche nos écarts de conduite et qui parvient même, lors d'une fin mémorable, à nous éliminer, faute d'avoir su préserver la cohérence de son œuvre. Cependant, le joueur ne fait en définitive que contrarier certaines trames du récit. Le jeu de Galactic Cafe a intégré la dimension destructive du briseur de jeu, pour en faire le cœur de son expérience, son mécanisme fondamental. The Stanley Parable est un jeu paradoxal, car ce simulacre de 
destruction est le seul jeu possible. Nous ne pouvons jouer qu'à détruire l'histoire d'un autre (celle du narrateur) - et c'est cette destruction même qui semble constituer, à proprement parler, notre jeu.

Ce paradoxe se comprend sans difficulté : toute l'ambition du jeu vidéo de Galactic Cafe est de rompre la magie qui nous tient habituellement en haleine, de mettre en scène cette illusion en tant qu'illusion et de donner au sabotage visuel et narratif auquel il nous invite une dimension critique qui s'étende à l'ensemble du dispositif vidéoludique. À cette fin, au lieu d'être associés comme dans les jeux qui suivent les canons de l'industrie, la narration et le fonctionnement du programme sont ici de parfaits ennemis. Le déroulement du programme (au service du seul mécanisme de jeu restant) assure le délitement de l'intrigue. Même le narrateur finit par se prêter au jeu. C'est lui qui, en véritable Dieu de l'univers de Stanley, l'emmène d'une salle à l'autre, le projette dans d'autres jeux vidéo, lui demande de noter son expérience, le fait atterrir dans un musée exposant l'envers du décor qui, dans un ultime retournement, détaille le processus de production du jeu lui-même.

En prenant la porte de droite, le joueur fait davantage que s'opposer au narrateur. La fonction de ce dernier est, nous l'avons dit, de personnifier le programme, d'incarner l'ordre des choses. En affrontant cette figure métaphorique, c'est donc au programme que le joueur tente de s'en prendre. C'est bien la fatalité informatique de l'expérience qu'il essaie vainement de conjurer. Seulement, pour citer un célèbre personnage de Diderot et, ajouterions-nous, dans les jeux vidéo davantage que dans la vie : « Tout est écrit là-haut ${ }^{21}$. » Il s'ensuit un retournement qui n'est pas sans évoquer la morale tirée d'une des histoires de ce fameux roman philosophique. Lorsque le maître tente de convaincre Jacques qu'être libre est agir selon son bon vouloir, le servant évoque la philosophie de son vieux capitaine :

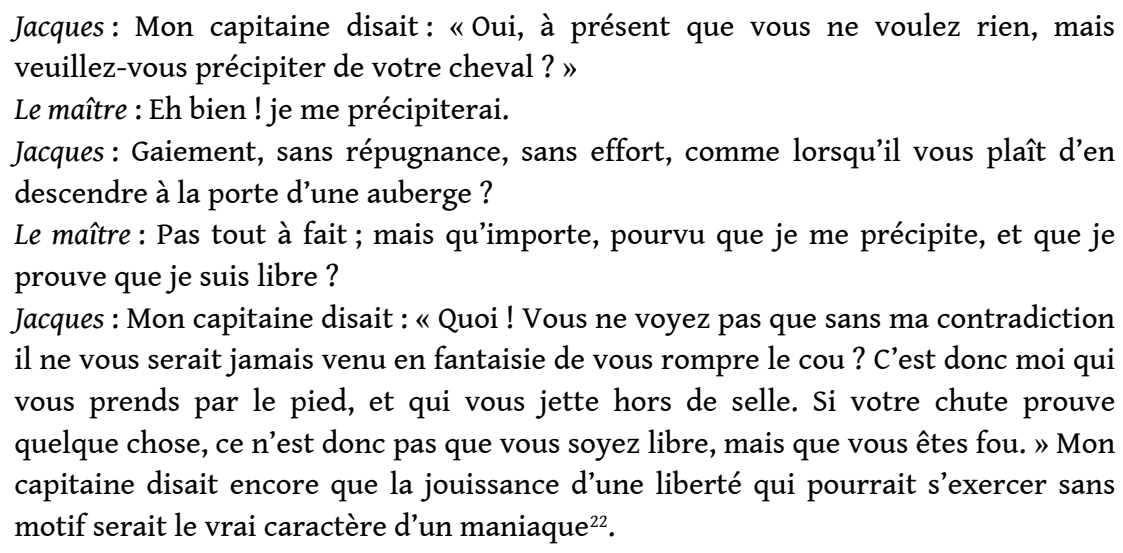

L'une des fins possibles reprend exactement les termes du problème tel qu'il est posé par Diderot : le jeu nous guide jusqu'à un escalier de plusieurs étages d'où Stanley, dans un geste suicidaire, doit se jeter plusieurs fois pour contrecarrer les plans du narrateur - quitte à y laisser sa «vie ». Le joueur choisit donc de faire mourir son personnage pour prouver sa liberté vis-à-vis de cette entité surplombante, qui le prie de renoncer à chaque nouvelle tentative; mais l'espace du jeu, l'aménagement programmé des situations ne laisse au joueur que cette porte de sortie. Si les prières du narrateur sont vaines, c'est bien, pour reprendre le mot de Diderot, le programme qui nous prend par la main et nous jette dans le vide.

La structure programmatique est inamovible. Elle est la condition technique de l'expérience et devient cette contradiction qui empêche finalement le déploiement de 
notre liberté hors des voies prévues. Mieux, c'est cette structure qui nous incite à investir notre liberté dans ce refus. Donc, quand nous contredisons le narrateur, nous obéissons au programme. L'insoumission à l'intrigue et au narrateur est une soumission au programme et à son ordre prétabli. Dans The Stanley Parable, l'insoumission est davantage que la soumission poursuivie par d'autres moyens; elle est la soumission véritable au mécanisme du jeu, ou, pour reprendre une formule canonique, une négation du déterminisme dans sa propre sphère.

\section{Une certaine conception de la liberté}

C'est pourquoi, in fine, le narrateur et Stanley sont également impuissants. Ils ne sont que la manifestation du programme, qui organise un deadlock - une étreinte fatale qui pousse le joueur à recommencer éternellement la partie. Si le sujet humain tente inlassablement d'échapper au cours obligé des choses, il est inévitablement rattrapé par le jeu. Les multiples fins ne sont que des détours pour arriver à la même conclusion, qui donne toute sa substance à la parabole : celle d'un homme qui n'a jamais quitté son travail et qui presse les touches de son clavier quand son écran le lui ordonne.

Le narrateur traite les jeux vidéo comme de simples fantaisies de Stanley :

Mais dans son esprit, ah, dans son esprit il peut prendre part à des aventures fantastiques! Derrière son bureau, Stanley rêvait d'expéditions endiablées dans l'inconnu, de découvertes fantastiques de nouveaux territoires! C'était merveilleux. Et chaque jour où il revenait travailler était un rappel qu'il ne vivrait jamais rien de tout cela ${ }^{23}$.

Bien évidemment, «En réalité, il ne fait qu'appuyer sur les mêmes boutons. Rien n’a changé ${ }^{4}$. »

La cause de cette condition peu enviable est aussitôt révélée :

Et j'essaie de lui dire que, dans ce monde, il ne sera jamais rien d'autre qu'un observateur, qu'autant qu'il reste ici, il se tue à petit feu. Mais il ne m'écoute pas, il ne s'arrête pas ${ }^{25}$ !

Le fait est que le jeu, en nous mettant dans le rôle de Stanley, nous réduit à l'état d'une monade infirme incapable de prendre une décision qui ait quelque conséquence. Le rapport collectif à la liberté est absolument laissé pour compte. C'est ici la seule capacité à choisir qui semble sous-tendre la conception que les développeurs de Galactic Cafe se font de la liberté. Elle est ici une qualité du sujet, sa capacité à s'orienter dans le monde et à se définir à travers chaque décision prise. Le sujet, pour n'être pas le jouet des circonstances, doit pouvoir choisir, c'est-à-dire s'affirmer de façon singulière en face d'elles. C'est ainsi qu'il peut écrire son histoire. Or, l'expérience vidéoludique repose sur la domination inverse, celle de l'objet, du programme, qui configure a priori toutes les réponses possibles.

Il n'existe pas dans le jeu vidéo, comme chez $\mathrm{Kant}^{26}$, une liberté transcendantale qui puisse extirper le sujet de l'hétéronomie de la nature. L'hétéronomie du programme est plus implacable que celle du monde sensible et aucune obéissance consentie par la raison à une loi morale universelle ne permet d'échapper au déterminisme. Cela vient du fait que la dimension empirique du jeu vidéo est tout entière tenue par la dimension intelligible du code. Si, chez Kant, le monde de l'intelligible est le garant d'une liberté vis-à-vis du monde empirique, comme la raison l'est vis-à-vis des désirs, dans l'univers du jeu vidéo, au contraire, l'expérience concrète est absolument conditionnée par une 
structure intelligible indépendante du sujet. La seule raison qui vaille est celle du programme. Il est le seul impératif catégorique. L'autonomie est impossible.

Le jeu vidéo s'emploie bien davantage à réaliser la conception spinoziste de la liberté, en détournant dans la pratique la célèbre maxime de l'Éthique, selon laquelle les joueurs se croient libres car ils sont conscients de leurs actions et ignorants des causes par où ils sont déterminés. The Stanley Parable se défait de la cohérence narrative qui masque habituellement le déterminisme du programme : mieux, le jeu se sert de son intrigue fragmentée pour évoquer, à chaque fin avortée et par ses éternels recommencements, la structure inaltérable de l'expérience. Le voile de la liberté apparente qu'offrent les autres jeux vidéo est arraché. Au lieu d'un libre-arbitre au sein d'une expérience parfaitement conditionnée, le conditionnement se manifeste ici dans toute sa force. En définitive, The Stanley Parable est le cauchemar de l'individualisme, son effroi devant la contrainte impersonnelle du programme, devenu fable.

\section{BIBLIOGRAPHIE}

Bera Tristan, «L'identité dans The Talos Principle, The Stanley Parable et The Swapper », Conserveries mémorielles, $\mathrm{n}^{\circ} 23,2018$.

Berlin Isaiah, Éloge de la liberté, trad. de l'anglais J. Carnaud et J. Lahana, Paris, Calmann-Lévy, coll. « Liberté de l'esprit », 1988.

Diderot Denis, Jacques le fataliste et son maître [1796], La Bibliothèque électronique du Québec [en ligne]. URL : https://beq.ebooksgratuits.com/vents/Diderot-Jacques.pdf [consulté le 18/07/2020]

Hegel Georg W. F., La Phénoménologie de l'esprit [1807], t. I, trad. de l'allemand J. Hyppolite, Paris, Aubier Montaigne, coll. «Philosophie de l'esprit », 1939.

Hoare Douglas, Le Jeu vidéo ou l'aliénation heureuse, Paris, Post-éditions, 2019.

Huizinga Johan, Homo Ludens, essai sur la fonction sociale du jeu, Paris, Gallimard, coll. « Les Essais », 1951.

Kant Emmanuel, Critique de la raison pratique [1788], éd. établie sous la dir. de F. Alquié, trad. de l'allemand par L. Ferry et H. Wismann, Paris, Gallimard, 1989.

Littré Émile, Dictionnaire de la langue française [en ligne], Paris, Louis Hachette, 1873-1877. URL : https://www.littre.org [consulté le 15/04/2020]

Marti Marc, « La narrativité vidéoludique : une question narratologique », Cahiers de Narratologie, $\mathrm{n}^{\circ} 27,2014$.

Quiniou Yvon, «Pour une actualisation du concept d'aliénation », Actuel Marx, n 39, 2006.

Ryan Marie-Laure et Rebreyend Anne-Laure, « Des jeux narratifs aux fictions ludiques. Vers une poétique de la narration interactive », Nouvelle revue d'esthétique, $\mathrm{n}^{\circ} 11,2013$, p. 37-50.

Spinoza Baruch, L'Éthique [1677], introduction et trad. du latin R. Caillois, Paris, Gallimard, coll. « Folio. Essais », 1994. 
Todorov Tzvetan, « Les catégories du récit littéraire », Communications, $\mathrm{n}^{\circ}$ 8, 1966, p. 125-151.

Valéry Paul, « Variété. Théorie poétique et esthétique. Première leçon du cours de poétique », in Euvres, t. I, éd. établie et annotée par J. Hytier, Paris, Gallimard, coll. « Bibliothèque de la

Pléiade », 1957.

\section{NOTES}

1. Les jeux d'aventure, les first-person shooters (FPS) et autres jeux de rôles (role-playing games ou RPG) en ont fait leur spécialité. Pour une classification des jeux vidéo en fonction de l'importance que prend chez eux le récit, voir l'article de Marc Marti : «La narrativité vidéoludique : une question narratologique », Cahiers de Narratologie, $\mathrm{n}^{\circ} 27,2014$.

2. Voir Marie-Laure Ryan et Anne-Laure Rebreyend, «Des jeux narratifs aux fictions ludiques. Vers une poétique de la narration interactive ", Nouvelle revue d'esthétique, $\mathrm{n}^{\circ} 11,2013$, p. 37-50.

3. Id.

4. Distinction établie par Paul Valéry. Voir Paul Valéry, «Variété. Théorie poétique et esthétique. Première leçon du cours de poétique ", in Euvres, t. I, Paris, Gallimard, coll. «Bibliothèque de la Pléiade », 1957.

5. Galactic Cafe, The Stanley Parable, 2013. Nous traduisons «This is the story of a man named Stanley. "

6. L'absence d'identité du protagoniste est l'un des traits saillants du jeu. Pour aller plus loin, voir Tristan Bera, "L'identité dans The Talos Principle, The Stanley Parable et The Swapper ", Conserveries mémorielles, $\mathrm{n}^{\circ} 23,2018$.

7. Ibid.

8. Tzvetan Todorov, « Les catégories du récit littéraire ", Communications, $\mathrm{n}^{\circ}$ 8, 1966, p. 141.

9. Yvon Quiniou, «Pour une actualisation du concept d'aliénation », Actuel Marx, $\mathrm{n}^{\circ}$ 39, 2006, p. 71-88.

10. Tzvetan Todorov, op. cit., p. 142.

11. Sur les concepts de liberté positive et négative, voir Isaiah Berlin, Éloge de la liberté, Paris, Calmann-Lévy, coll. « Liberté de l'esprit », 1988.

12. Galactic Cafe, The Stanley Parable, 2013. Nous traduisons « He [...] unshackled himself from someone else's command. »

13. Ibid. Nous traduisons «This was exactly the way, right now, that things were meant to happen. And Stanley was happy. »

14. Émile Littré, Dictionnaire de la langue française [en ligne], Paris, Louis Hachette, 1873-1877. URL : https://www.littre.org [consulté le 15/04/2020]

15. Douglas Hoare, Le Jeu vidéo ou l'aliénation heureuse, Paris, Post-éditions, 2019, p. 20.

16. Voir Isaiah Berlin, op. cit.

17. Voir Georg W. F. Hegel, La Phénoménologie de l'esprit [1807], t. I, Paris, Aubier Montaigne, coll. «Philosophie de l'esprit », 1939, p. 161-162.

18. Mojang Studios, Minecraft, 2009.

19. Valve, Portal, 2007.

20. Johan Huizinga, Homo Ludens, essai sur la fonction sociale du jeu, Paris, Gallimard, coll. «Les Essais », 1951, p. 32.

21. Denis Diderot, Jacques le fataliste et son maître [1796], La Bibliothèque électronique du Québec [en ligne]. URL: https://beq.ebooksgratuits.com/vents/Diderot-Jacques.pdf [consulté le 18/07/2020]

22. Ibid., p. 483-484. 
23. Galactic Cafe, The Stanley Parable, 2013. Nous traduisons «But in his mind, ah, in his mind he can go on fantastic adventures! From behind his desk, Stanley dreamed of wild expeditions into the unknown, fantastic discoveries of new lands! It was wonderful! And each day that he returned to work was a reminder that none of it would ever happen to him ».

24. Id. Nous traduisons "[in] reality, all he's doing is pushing the same buttons. Nothing has changed ».

25. Id. Nous traduisons « And I'm trying to tell him this, that in this world, he cannot be anything but an observer, that as long as he remains here, he is slowly killing himself. But he won't listen to me, he won't stop! ».

26. Emmanuel Kant, Critique de la raison pratique [1788], Paris, Gallimard, 1989.

\section{RÉSUMÉS}

L'expérience interactive du jeu vidéo permet à tout joueur de concourir à la narration de sa partie. D'un jeu vidéo à l'autre, le dispositif lui laisse une certaine latitude dans la mise en scène des événements et dans leur déroulement. En tant que " co-créateur de son expérience ludique ", le joueur entre dans The Stanley Parable (Galactic Cafe, 2013) en conflit ouvert avec un personnage singulier : le narrateur. Figure allégorique s'il en est, ce personnage de narrateur - simple voix hors champ dictant le cours de l'action - est le symbole d'une contrainte structurelle qui s'exerce habituellement de manière impersonnelle. Alors que la plupart des jeux vidéo ont tendance à faire passer le fil d'un scénario pré-écrit pour une suite de décisions appartenant au joueur seul, la contradiction est ici tendue à l'extrême : la contrainte de la narration est personnalisée et l'on peut lui désobéir. Lorsque Stanley, avatar du joueur, arrive devant deux portes, la voix lui intime de prendre celle de gauche. Libre à lui de prendre celle de droite. Une lutte s'instaure alors entre le joueur et le narrateur pour le contrôle de l'histoire.

Cette lutte entre narrateur et avatar, au cœur de la mécanique ludique de The Stanley Parable, nous semble questionner la place du joueur dans le dispositif du jeu vidéo. À l'instar des films de la Nouvelle Vague, le jeu de Galactic Cafe parodie les codes et les canons de l'industrie et va jusqu'à les déconstruire pour proposer une expérience singulière de jeu. Cette lutte entre le joueur et le narrateur, explicitée par la mise en scène, n'est-elle pas à l'image de l'ambivalence entre poïèse et esthèse qui structure toute expérience vidéoludique ? N'est-elle pas l'expression imagée d'une lutte intérieure que connaît tout joueur?

\section{INDEX}

Mots-clés : Stanley Parable, joueur, narrateur, parabole, jeux vidéo, vidéoludique 


\section{AUTEUR}

\section{DOUGLAS HOARE}

Douglas Hoare est docteur en esthétique, diplômé de l'université Paris 8 , où il a soutenu une thèse portant sur les jeux vidéo. Il a publié chez Post-éditions en septembre 2019 un essai intitulé Le jeu vidéo ou l'aliénation heureuse. 